\title{
A APLICAÇÃO DA TEORIA DAS RESTRIÇÕES (TOC) E DA SIMULAÇÃO NA GESTÃO DA CAPACIDADE DE ATENDIMENTO EM HOSPITAL DE EMERGÊNCIA
}

\section{THE THEORY OF CONSTRAINTS AND THE SIMULATION APPLIED IN THE CAPACITY MANAGEMENT OF ATTENDANCE IN EMERGENCY HOSPITAL}

\author{
Francisco Santos Sabbadini \\ Doutorando em Engenharia de Produção - COPPE/UFRJ \\ Professor Auxiliar, MSc \\ Universidade Estácio de Sá - Ciências Sociais \\ Rua Zenaide Vilela, s/n - Resende - RJ - 27510-030 \\ (24) 3355-4489 - e-mail: sabbadini01@yahoo.com.br

\section{Antônio Augusto Gonçalves \\ Professor Auxiliar, DSc \\ Universidade Estácio de Sá} \\ Programa de Pós-Graduação em Administração \\ E Desenvolvimento Empresarial
}

Av. Presidente Vargas, 642, $22^{\circ}$ andar - Centro - Rio de Janeiro - RJ - 20071-001

(21) 2206-9743 - e-mail: antonio.gonçalves@estacio.br

\section{Mário Jorge Ferreira de Oliveira}

Professor Adjunto, $\mathrm{PhD}$

Universidade Federal do Rio de Janeiro

COPPE

Cidade Universitária - C. T. - Blc. F - S. 105 - Rio de Janeiro - RJ - 21945-970

(21) 2562-8244 - e-mail: Mario_jo@ @ep.ufrj.br 


\title{
RESUMO
}

No ambiente hospitalar os serviços envolvem processos complexos, de alto risco e custos elevados, particularmente numa unidade de emergência hospitalar. Essa é uma das áreas mais complexas e críticas de um hospital, onde a rapidez no atendimento é fundamental. A agilidade no encaminhamento dos pacientes que necessitam de cuidados médicos é fator crítico de sucesso para redução de seqüelas e nas chances de restabelecimento. Nesse contexto, torna-se importante a gestão da capacidade de atendimento, a adequada alocação e o dimensionamento dos recursos. Este artigo objetiva investigar alternativas que agilizem o atendimento a pacientes. Neste contexto são aplicados princípios de gestão da capacidade de serviços, feita a análise do fluxo de tratamento a pacientes, utilizada a teoria das restrições na identificação de gargalos e desenvolvido um modelo de simulação a eventos discretos. A partir desse ferramental teórico elaborou-se uma proposta de melhoria para a redução do tempo entre o registro do paciente de urgência e o início do seu efetivo tratamento.

Palavras-chave: Teoria das restrições (TOC) , Gestão da capacidade de serviços, Simulação, Hospital, serviços de saúde.

\begin{abstract}
The hospital environment services involves of high risk, complex processes and costs, especially in a unit of hospital emergency. This is one of the most complex and critical areas of a hospital, where the service efficiency is fundamental. The agility in guiding the patients who need medical care is a critical factor for successful reduction of damage and for possibilities of recovery. The management of service capacity becomes important, as well as the adjusted allocation and sizing of resources. The aim of this thesis is to investigate alternative policies that speed up the attendance the patients. The principles of capacity management of services are applied and treatment flow the patients is analyzed. The theory of the Constraints is used to identify bottlenecks. A simulation model of unremarkable events is made. Based on these theoretical tools, a proposal of improvement for the reduction of the time between the register of the urgency patient and the beginning of his/her effective treatment has been drawn up.
\end{abstract}

Key-words: Theory of Constraints, Capacity management of services, Simulation, Hospital, Health services

\section{INTRODUÇÃO}

No atendimento de emergência o tempo é um fator crucial. A meta desse setor é a avaliação rápida, a estabilização, o tratamento e pronta admissão do paciente ao hospital. Quanto mais eficazes forem esses serviços, maiores as chances de restabelecimento da saúde do paciente. 
No atual ambiente econômico, com sérias restrições orçamentárias, o controle de recursos escassos aliado à eficiência em sua utilização se torna crítico. Administradores da área de saúde lidam com o desafio de conduzir organizações de grande complexidade e que possuem processos de forte interatividade, aonde a gestão da capacidade de atendimento desempenha um papel estratégico que envolve recursos escassos e de alto custo.

Neste cenário é fundamental o ajuste da capacidade dos serviços médicos oferecidos à demanda, identificando os recursos onde à alteração da capacidade vai gerar o maior benefício. Para tal estratégia, utilizou-se o processo de focalização da teoria das restrições (TOC), identificando os fatores limitantes do sistema.

Apesar da teoria das restrições ter nascido no setor industrial, sua filosofia pode ser aplicada em diferentes tipos de organizações, já existindo aplicações da TOC na área de saúde. Na Inglaterra esta teoria foi aplicada com sucesso na redução das longas listas de espera que são administradas pelo sistema nacional de saúde (PHIPPS, 1999).

Aplicando os princípios da TOC, foi realizada uma análise do fluxo de tratamento e identificada a restrição no processo de cirurgias. Constatou-se que o número de pacientes internados para intervenção cirúrgica era superior à capacidade de atendimento dos médicos. Estabeleceu-se um sistema de gerenciamentos das filas e reduziu-se a programação de pacientes internados para cirurgias de 11 para 6. Como resultado obteve-se uma redução no número de cirurgias de emergência e também um aumento de $16 \%$ na capacidade de atendimento do sistema.

$\mathrm{Na}$ área do tratamento de câncer foi realizado um estudo interessante no setor de quimioterapia de uma clínica oncológica (KERSHAW, 2000). Segundo este autor, Enquanto no ambiente de manufatura se busca uma maior produtividade, na área hospitalar a meta é atender com qualidade o maior número de pacientes. Similarmente, uma restrição pode ser considerada como um dos processos que compõem o fluxo de tratamento de pacientes. Uma diferença óbvia entre o ambiente industrial e o de saúde é que, diferentemente da manufatura, o que é tratado em um hospital é o ser humano. Nesta situação, a aplicação dos princípios da teoria das restrições é acrescida de novos desafios. Um exemplo disto é a importância da satisfação do paciente no processo de tratamento e sua percepção em relação à qualidade dos serviços prestados.

Nesta mesma área, pode-se citar o trabalho pioneiro no INCA - Instituto Nacional do Câncer, no Rio de Janeiro, no qual o processo de focalização da teoria das restrições foi 
utilizado para identificar e explorar gargalos no fluxo de tratamento de pacientes (GONÇALVES, 2004).

A restrição foi identificada no processo de diagnóstico, especificamente nos exames de imagem. A partir dessa constatação, foram definidas ações no sentido de explorar e subordinar todo os recursos à restrição identificada. Como resultado, obteve-se uma redução de $25 \%$ de tempo entre o registro do paciente e o início do tratamento efetivo.

Em unidade de emergência hospitalar pode-se citar o estudo realizado no Sheba Medical Center em Israel, no sentido de reduzir o tempo de espera por atendimento naquela organização (ROTSTEIN et al, 2002). O estudo partiu da hipótese de que as taxas de admissão têm efeito sobre o prolongamento do tempo individual dos pacientes. Baseado na Teoria das Restrições foi realizado um experimento que analisou a adição de mais um médico para aliviar a pressão na emergência. Os resultados demonstraram que o tempo de permanência caiu em 6,61 minutos para a faixa de 80 a 119 pacientes admitidos no setor, bem como houve aumento da sua satisfação com o serviço.

Associada a TOC utilizou-se a simulação como ferramenta de apoio. Segundo De Oliveira (1994) a simulação consiste num conjunto de entidades representativas de objetos reais entre os quais são estabelecidos relacionamentos.

De acordo com Banks e Carson (1984) a simulação é uma técnica que imita operações de processos reais num dado período de tempo, sendo utilizada como ferramenta analítica e instrumento de apoio à tomada de decisões.

O objetivo deste trabalho é a apresentação de uma abordagem inovadora de gestão da capacidade de atendimento em hospital de emergência, aplicando os conceitos da teoria das restrições na identificação de gargalos no fluxo de tratamento e da simulação computacional para analisar as melhores alternativas de acesso e utilização de recursos humanos disponíveis, no sentido de agilizar o atendimento a pacientes e reduzir as filas de espera.

Quanto à metodologia utilizou-se o estudo de caso único no Hospital Municipal de Emergência Henrique Sérgio Gregori (HMEHSG), conforme Yin (2001). A pesquisa é caracterizada como aplicada, de campo e bibliográfica (VERGARA, 2005).

\section{2- SERVIÇOS E SAÚDE}

As atividades de serviço ganham importância cada vez maior na sociedade contemporânea, e passam a demandar formas mais eficientes e eficazes de gestão da 
capacidade das operações. Os serviços possuem características diferenciadoras em relação à manufatura, a simultaneidade entre sua produção e o seu consumo, a intangibilidade e a interação com o cliente, o qual participa do processo de produção.

Segundo Fitzsimmons e Fitzsimmons (2000) devido à intangibilidade dos serviços sua capacidade é perecível com o tempo. Se o serviço não for utilizado, sua capacidade instalada é perdida definitivamente. Em relação à simultaneidade como a produção e consumo ocorrem ao mesmo tempo os serviços não podem ser estocados.

A interação com o cliente, que participa no processo de serviço, possibilita a ocorrência de variações no atendimento, no desempenho e na percepção deste em relação ao serviço prestado (CORREA E GIANESI, 1994).

No caso das unidades de emergência as demandas dos pacientes exibem variações consideráveis e não existe a opção de formar estoques para absorver estas flutuações. O comportamento cíclico torna ainda mais importante à adequada alocação e utilização dos recursos.

As operações são de elevada complexidade e possuem forte interação. No ambiente hospitalar, há uma forte interdependência entre os diversos processos internos. Pode ocorrer que algumas áreas da organização trabalhem abaixo da capacidade, gerando ociosidade, e outras no seu limite máximo, tornando-se uma restrição ao seu desempenho como um todo.

\section{3- ESTUDO DE CASO}

O HMEHSG é um dos componentes do sistema de saúde do município de Resende, RJ, e integra o Sistema Único de Saúde (SUS), com a finalidade de prestar atendimento integral a pacientes em estado de urgência ou emergência.

Devido à eficácia na prestação de atendimento de casos dessa natureza, além da sua infra-estrutura e localização geográfica, o HMEHSG tornou-se ao longo dos anos uma referência neste tipo de serviço para o município de Resende, sendo hospital de referência no atendimento de acidentes ocorridos na Rodovia Presidente Dutra.

O serviço de emergência do hospital funciona 24 horas, todos os dias, atendendo diariamente uma média de 192 pacientes. Em de 2004 foram registrados 70.480 atendimentos.

A direção do hospital verificou que ao longo dos últimos anos a sua capacidade de atendimento tem sido inferior à demanda, verificando-se com freqüência o congestionamento Revista Produção Online, Florianópolis, v.6, n.3,p.55, set./dez., 2006 
na unidade de emergência e a formação de longas filas de espera, que geram desconforto aos pacientes e pressão sobre a equipe médica.

Uma constatação importante, referente ao perfil dos pacientes, é que a maioria dos que procuram a unidade de emergência não necessita de atendimento imediato, conforme mostra a figura 1, prejudicando os pacientes com casos de urgência e de emergência.

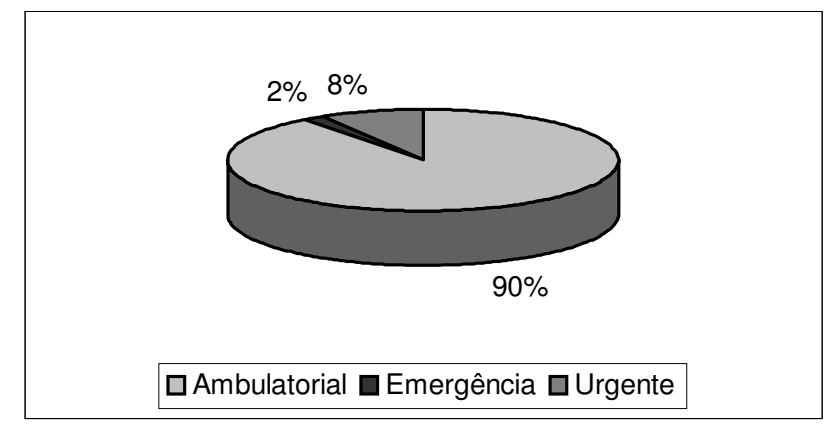

Figura 1 - Percentual de atendimentos por perfil de paciente

Fonte: Sabbadini (2005, p. 118).

Neste contexto a teoria das restrições mostrou-se adequada para a caracterização e tratamento do problema em questão, uma vez que o objetivo principal é agilizar o fluxo de tratamento de pacientes, identificando e gerenciando gargalos.

Para esse fim, foi elaborado o fluxo de tratamento a pacientes no HMEHSG desde a triagem até o tratamento. Toda a elaboração do fluxo foi realizada in loco com o acompanhamento do médico chefe da emergência e posteriormente validado por outros membros do corpo clínico, envolvendo médicos e enfermeiros daquela unidade.

\section{4- A TEORIA DAS RESTRIÇÕES E A SIMULAÇÃO}

Segundo a TOC, toda empresa é um sistema tangível cujo desempenho vai depender da forma como interagem seus diferentes processos. A restrição pode ser identificada pela existência de filas de espera ou por tempos de processamento longos (GOLDRATT, 1990).

Existem duas premissas em sua abordagem. A primeira é considerar a empresa como um sistema cujo resultado depende da forma como diferentes processos interagem. A segunda é a de que uma restrição é qualquer fator que limite o sistema de atingir sua meta. Sendo 
assim, todo sistema deve ter pelo menos uma restrição ou fator limitante. Uma restrição não é boa nem má. Ela existe e deve ser gerenciada (GOLDRATT, 1990; 1994).

Segundo Schragenheim (1998), o enfoque científico da TOC é baseado em algumas hipóteses e um conjunto de desenvolvimentos logicamente derivados dessas hipóteses, das quais três conceitos chave são: Toda organização tem uma meta a alcançar, sendo que ela é mais do que a soma da suas partes e o seu desempenho é restringido por poucas variáveis. Neste sentido de acordo com Cox III (2002), esse modelo de gerenciamento é uma abordagem que considera o papel da restrição na determinação dos resultados de um sistema o que permite o aprimoramento contínuo das operações pela coordenação do fluxo através dos recursos chaves das instalações.

$\mathrm{Na}$ teoria das restrições o processo de focalização em cinco etapas é uma metodologia que possibilita determinar como explorar os gargalos para obter melhorias no desempenho da organização. Segundo Goldratt (1990) os passos são os seguintes: 1) Identificar a restrição do sistema; 2) Decidir como explorar a restrição; 3) Subordinar todos os recursos não restritivos as restrições; 4) Elevar a restrição e 5) Se a restrição se deslocar não permitir que a inércia se instale e retornar ao passo 1.

O processo de melhoria contínua pode ser identificado em metodologias como o processo de focalização que consiste em focalizar a meta do sistema, determinar medidas de melhoria, identificar a restrição, gerenciá-la e subordinar todos os recursos a esta restrição. Ao reavaliar continuamente o sistema e seu processo se obtém um ciclo de melhoria, conforme se pode observar na figura 2 .

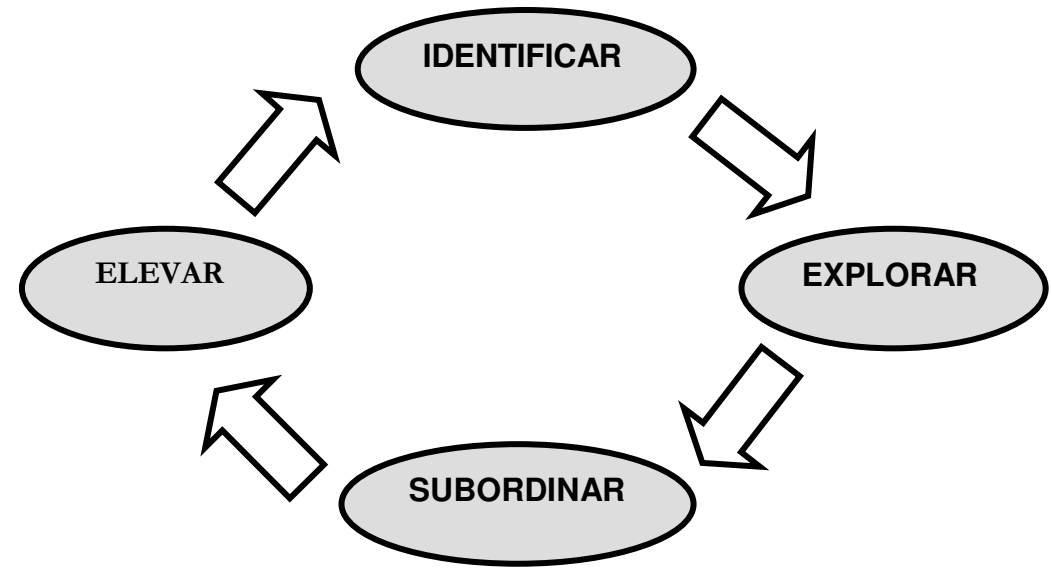

Figura 2 - Ciclo de melhoria contínua pela metodologia dos cinco passos 
Fonte: Sabbadini (2005, p. 65).

As restrições devem ser identificadas, porque são elas que vão determinar o desempenho do sistema como um todo. Uma gargalo pode ser identificado por longas filas de espera ou pelo maior tempo de processamento.

A figura 3 exemplifica o processo de identificação de uma restrição num sistema de saúde. Considera-se um fluxo hipotético de pacientes composto por quatro processos interdependentes: recepção, triagem, diagnóstico e tratamento.

Cada um desses processos possui recursos humanos, máquinas e equipamentos que permitem atender uma certa quantidade de pacientes, num determinado intervalo de tempo, em função da capacidade de cada um. No exemplo, o gargalo está no processo de diagnóstico, porque, de todos os quatro processos, é aquele que possui a menor taxa de atendimento: 26 pacientes por hora.

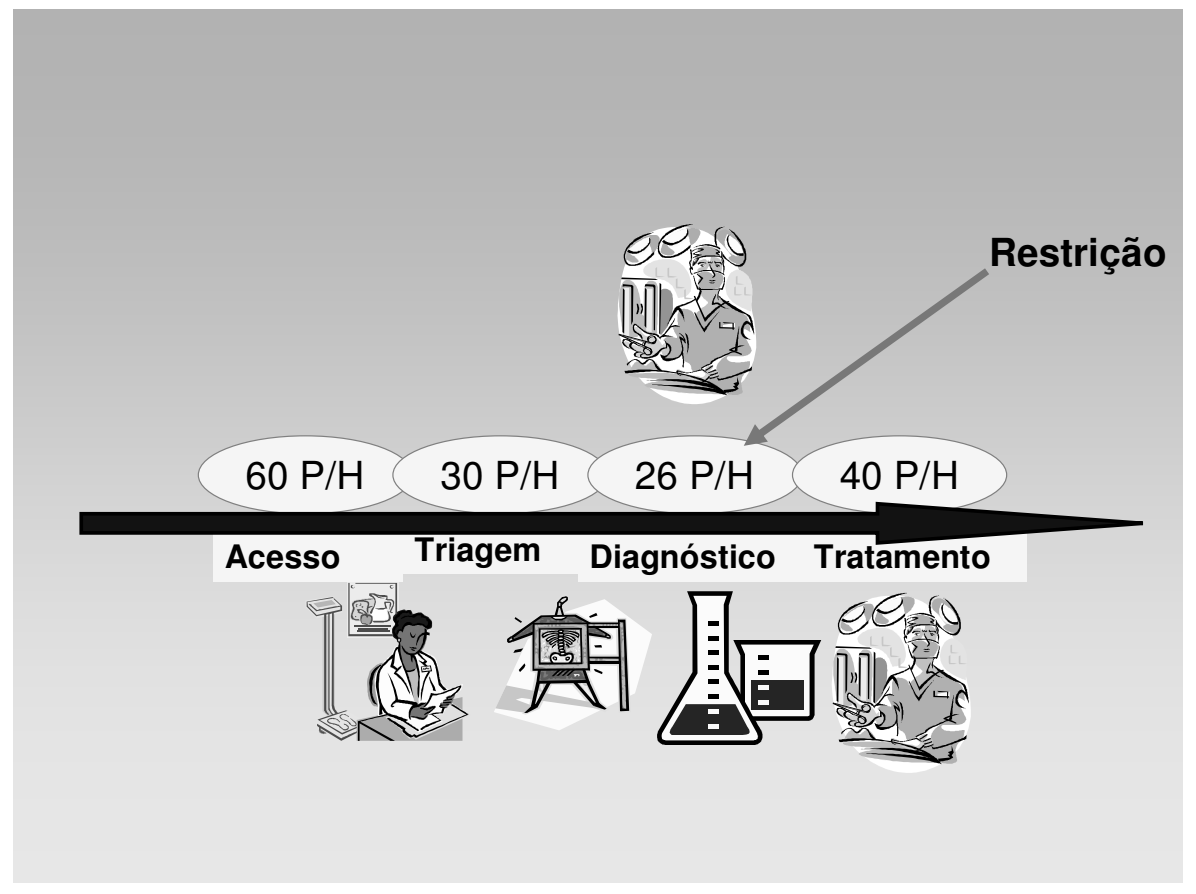

Figura 3 - Identificação de uma restrição

Fonte: Elaboração a partir de Goldratt, 1994. 
A partir dessa constatação, podem ser definidas ações no sentido de explorar e subordinar todos os recursos à restrição identificada e como resultado obter-se melhoria no fluxo de tratamento aos pacientes.

No HMEHSG o fluxo de pacientes, que é composto pelos processos de triagem, diagnóstico e tratamento foi analisado detalhadamente. São operações interdependentes, em que o desempenho de uma depende do resultado do imediatamente anterior. A figura 4 apresenta esquematicamente a sequiência dos serviços na unidade de emergência do hospital.

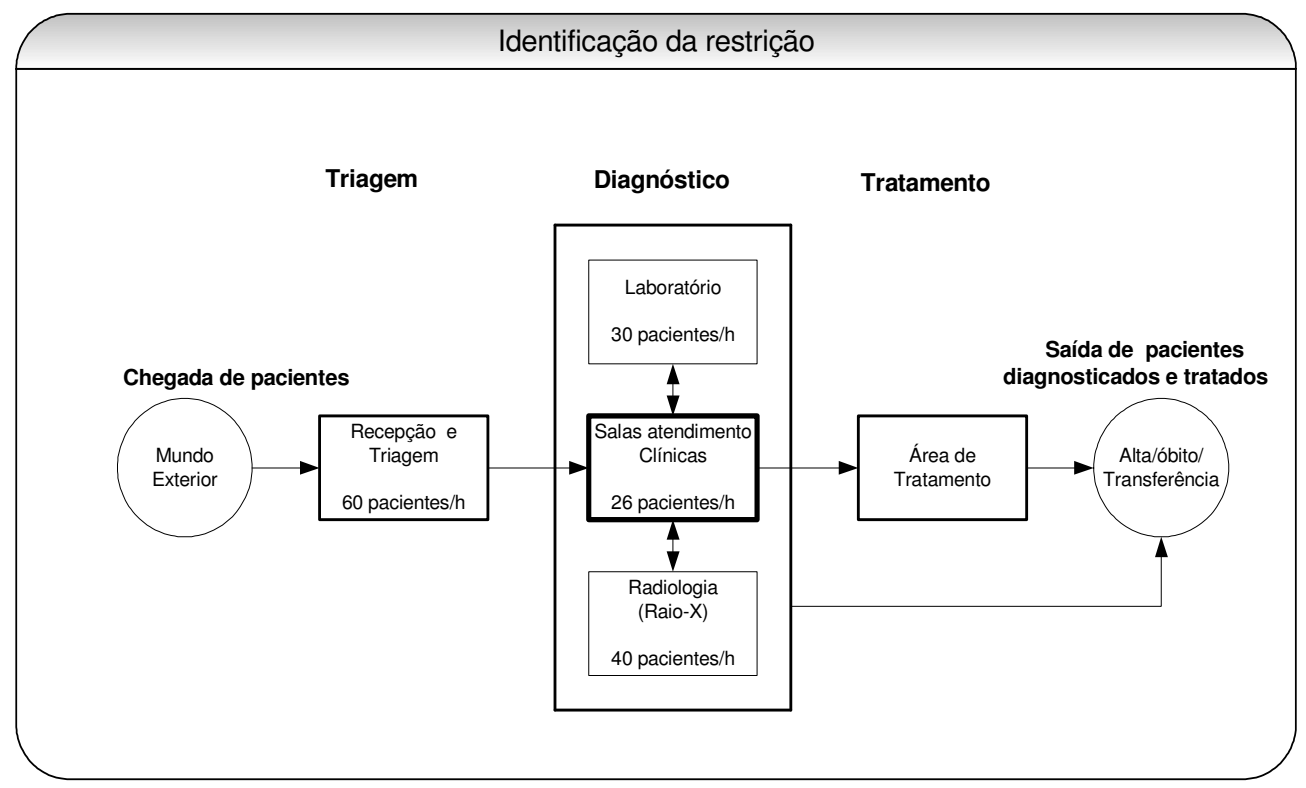

Figura 4 - Identificação da restrição

Fonte: Sabbadini (2005, p.143)

$\mathrm{Na}$ análise do fluxo de tratamento pode-se constatar o descompasso estrutural entre a oferta de recursos e a demanda, que resultam na formação de filas de espera, aumento do tempo de permanência de pacientes para o início do tratamento. Como se pode observar a menor taxa de atendimento está nas clínicas. O número de salas de disponíveis é uma restrição física que limita a taxa de atendimento pelos médicos.

Associada a essa restrição há pressão sobre os serviços e a equipe médica, que divide sua atenção entre a demanda de pacientes que chegam para o primeiro atendimento e a daqueles que retornam dos serviços de laboratório e radiologia, com os resultados dos exames. 
É importante destacar que a triagem dos pacientes no hospital é realizada pelo atendente da recepção, o qual não é habilitado para esta função. Concomitantemente há o número excessivo de pacientes ambulatoriais, que não necessitam de atendimento imediato, concorrendo com casos urgentes pelo atendimento dos médicos.

Conforme mencionado essa situação leva o paciente urgente a aguardar na fila de espera, sofrendo agravamento no seu quadro clínico, o qual somente será detectado efetivamente quanto este for atendido pelo médico. A partir dessa constatação definiu-se um modelo de simulação cujo escopo compreende os setores de pronto atendimento e prontosocorro da unidade de emergência, considerando as instalações, os recursos humanos, boletim de atendimento médico (BAM) e materiais relativos a cada processo analisado. A figura 5 apresenta o modelo conceitual. 


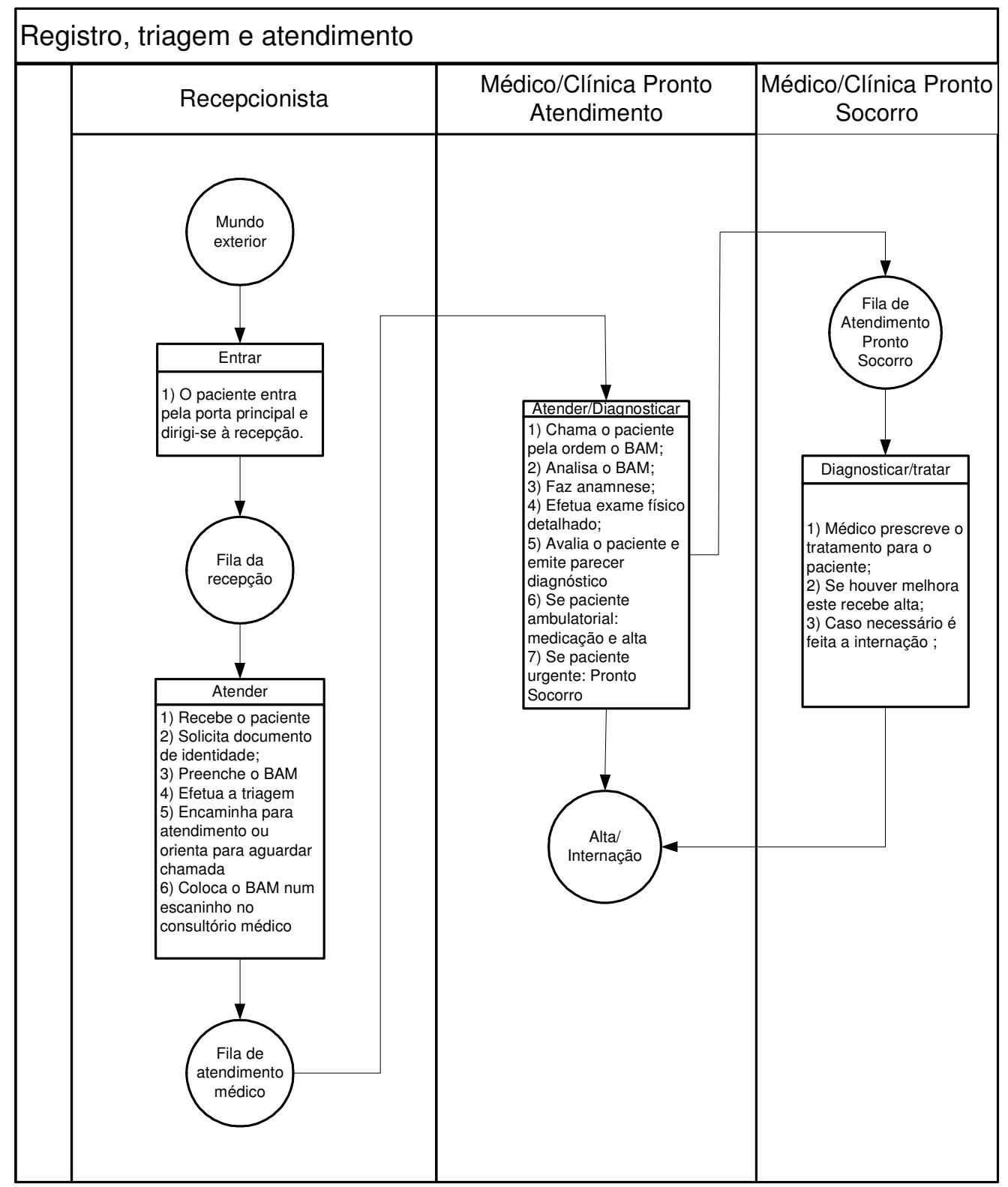

Figura 5 - Modelo conceitual na unidade de emergência do HMEHSG

Fonte: Sabbadini (2005, p. 149).

A partir do fluxo de tratamento a pacientes e do modelo conceitual foi desenvolvido o modelo computacional, mostrado na figura 6, com a utilização do software MedModel, versão 3.5, sendo a simulação terminal tendo o tempo de execução definido no relógio com início às $7 \mathrm{~h}$ e término às $23 \mathrm{~h}$. 


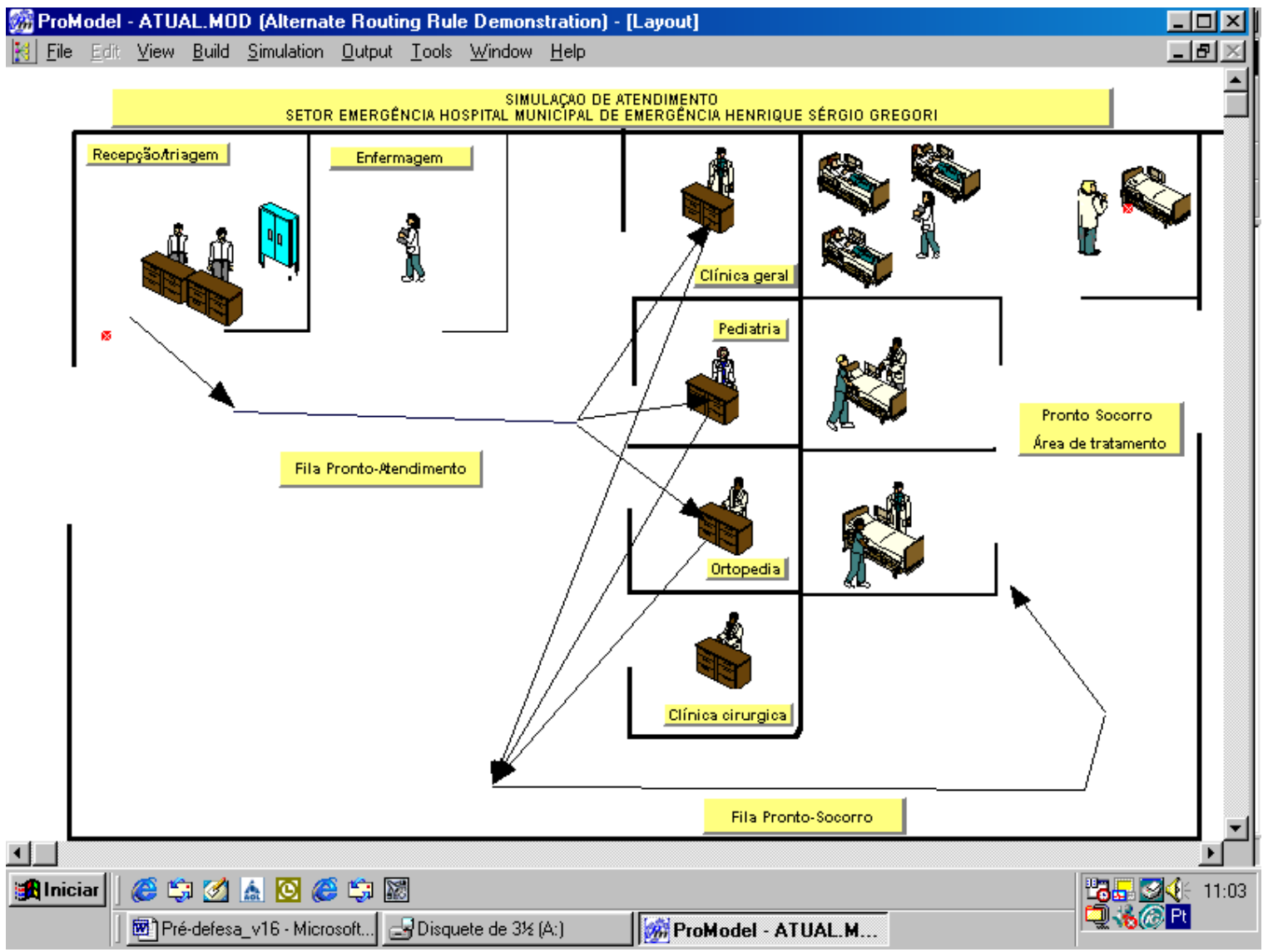

Figura 6 - Modelo de simulação

Fonte: Sabbadini (2005, p. 155).

O paciente chega à unidade de emergência, sendo recebido por uma das recepcionistas, que efetua o preenchimento e o registro do boletim de atendimento médico. Em seguida solicita ao paciente que aguarde na recepção.

O médico disponível recebe o paciente e inicia o atendimento. Faz a avaliação e emite o diagnóstico. Sendo um caso urgente é encaminhado para o Pronto-Socorro. Tratando-se de um paciente ambulatorial após a consulta este é liberado.

Segundo Rosseti et al (1999) devido à complexidade dos processos relacionados ao fluxo de pacientes e ao grande número de eventos aleatórios a coleta de dados numa unidade de emergência hospitalar deve ser efetuada em etapas.

A primeira etapa consistiu na elaboração e na análise do fluxo de tratamento a pacientes no HMEHSG que foi apresentado à direção do hospital e validado por esta. Na segunda etapa foram definidas as informações necessárias relacionadas ao modelo conceitual, que são 
descritas a seguir: a) taxa de chegada dos pacientes ambulatoriais e urgentes; e b) o tempo de atendimento em cada serviço (na recepção, na clínica geral, na pediatria, na ortopedia, na clínica cirúrgica e no pronto socorro).

$\mathrm{Na}$ terceira etapa foram efetivamente coletados os dados relativos às taxas de chegada de pacientes e aos tempos de atendimento em dias típicos de operação da unidade de emergência.

A animação do modelo computacional desenvolvido permitiu o seu completo entendimento pelos gestores e demais envolvidos no projeto, que o validaram como uma representação do sistema real.

Para garantir que o modelo estivesse construído corretamente adotaram-se os seguintes procedimentos no processo de verificação: 1) A seqüência lógica e os roteiros das entidades foram comparados com o fluxo de pacientes, confirmando sua correta representação no modelo computacional; 2) Foi utilizada a opção de rastreamento do MedModel, versão 3.5, o que permitiu o acompanhamento no modelo evento a evento, garantindo a representação acurada do sistema real; e 3) O experimento foi realizado diversas vezes. Os relatórios gerados foram comparados a situações conhecidas e analisados até que replicassem o modelo.

O foco da aplicação do modelo é na agilização do fluxo de pacientes urgentes pela melhoria no processo de triagem, tendo em vista que são os casos que demandam maior atenção naquele hospital, uma vez que os casos de emergência são prontamente atendidos, não havendo espera do paciente pelo atendimento médico.

No modelo proposto, o paciente chega à recepção sendo atendido pela recepcionista. Após cerca de 2 minutos, para preenchimento do boletim de atendimento médico (BAM), é encaminhado à triagem. A enfermeira, de posse do boletim de atendimento, faz a avaliação do paciente, para definir a severidade do caso e o seu encaminhamento. Sendo um paciente urgente é encaminhado para o pronto-socorro. Tratando-se de um paciente ambulatorial segue para o setor de pronto-atendimento, conforme se pode observar na figura 7. 


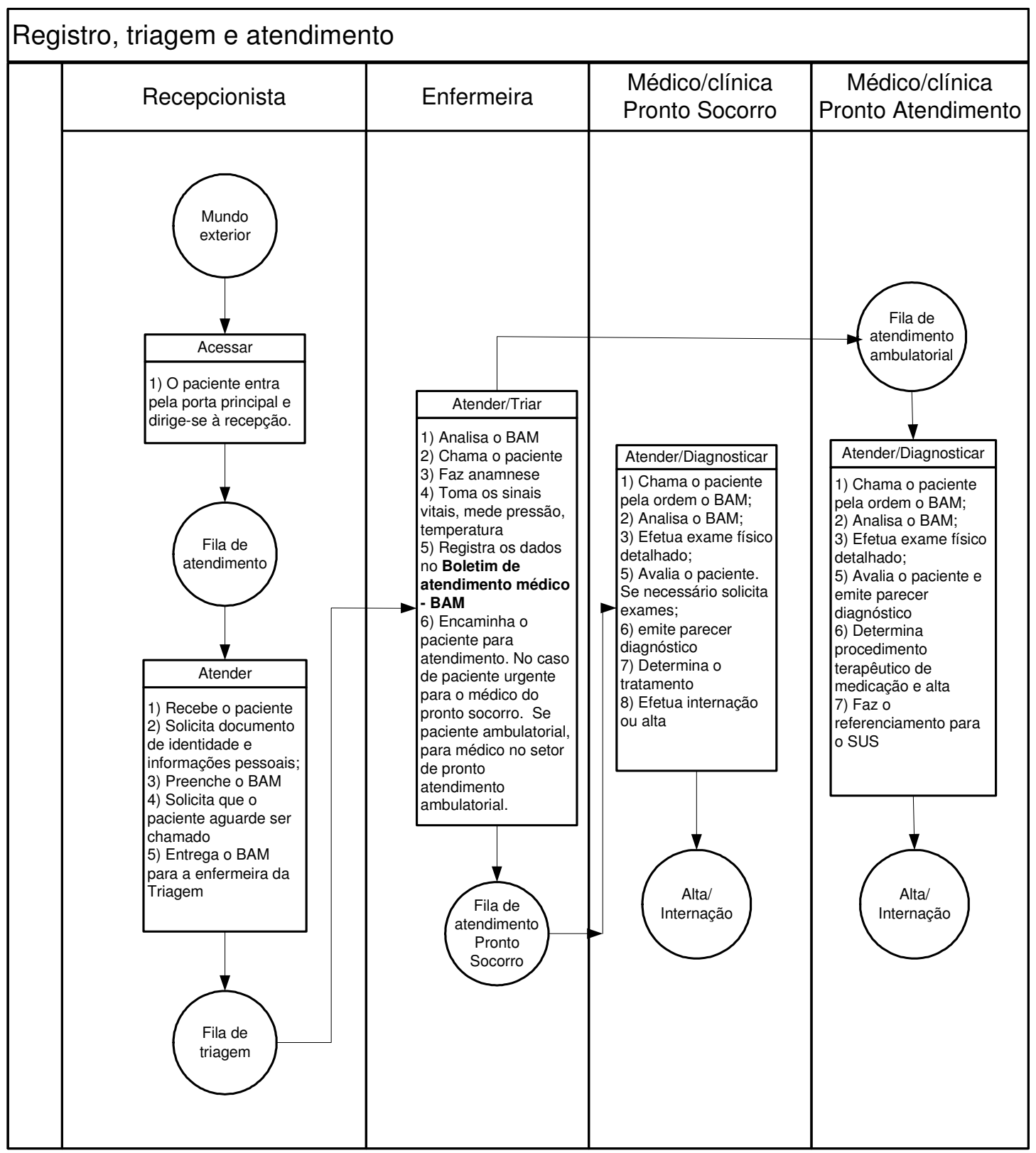

Figura 7 - Modelo conceitual proposto

Fonte: Sabbadini (2005, p. 162).

Elaborado o modelo conceitual proposto, foram efetuados os ajustes necessários no modelo computacional, de modo a refletir no simulador o comportamento deste, conforme se pode observar na figura 8. 


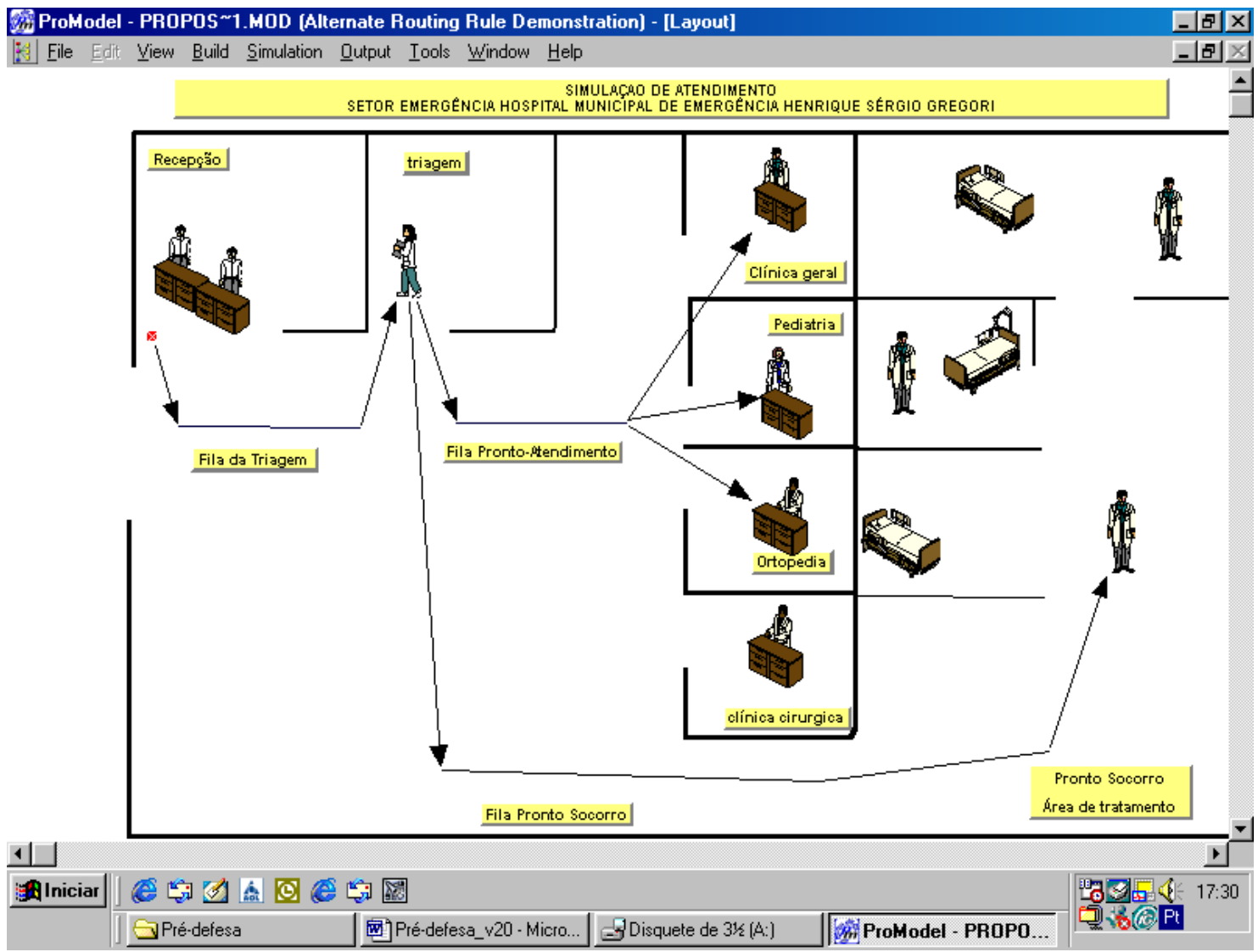

Figura 8 - Modelo computacional proposto

Fonte: Sabbadini (2005, p. 192)

Após a realização do experimento pode-se observar uma redução no tempo de espera do paciente com quadro urgente, conforme se observa na figura 9, pela melhoria do processo de triagem e pela realocação de médicos, em função da demanda em cada clínica. 


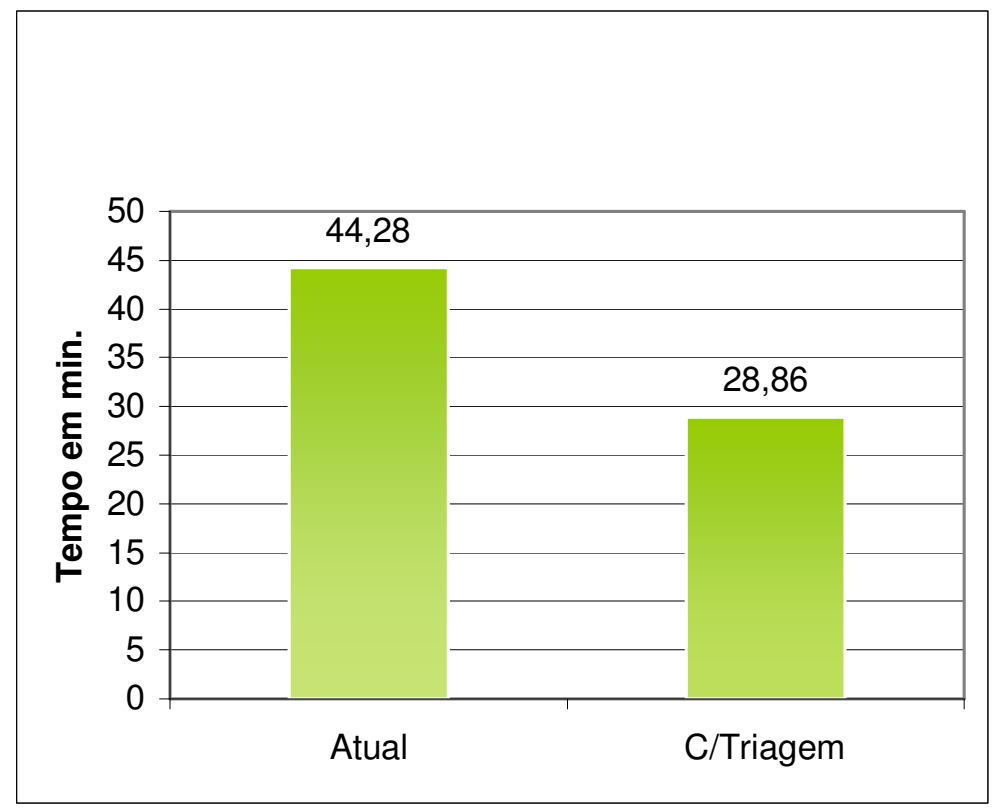

Figura 9 - Tempo médio de espera do paciente urgente por atendimento

Fonte: Sabbadini (2005, p. 171)

Um dos benefícios da simulação é a possibilidade de se verificar o comportamento do sistema e a partir daí definir onde se devem realizar as alterações e os investimentos para melhorar o atendimento.

Com a simulação pode-se visualizar a ocorrência da sobrecarga em determinadas clínicas e a ociosidade em outras, permitindo avaliar ações de curto prazo como a realocação de médicos nos horários de pico para atender a demanda dos pacientes e agilizar o fluxo de tratamento.

Em processos intensivos em recursos humanos a análise dos efeitos de alterações na sua configuração sobre o sistema é uma das questões que devem ser avaliadas. No caso da triagem a disponibilidade de enfermeiras disponíveis repercute diretamente nos resultados esperados. No HMEHSG há três enfermeiras alocadas à unidade de emergência.

$\mathrm{Na}$ figura 10 pode-se observar os efeitos sobre o tempo de espera do paciente urgente. Parte-se da situação atual, sem a triagem, e varia-se o número de enfermeiras alocadas ao setor considerando a triagem com 01 enfermeira, com 02 enfermeiras e com 03 enfermeiras. 


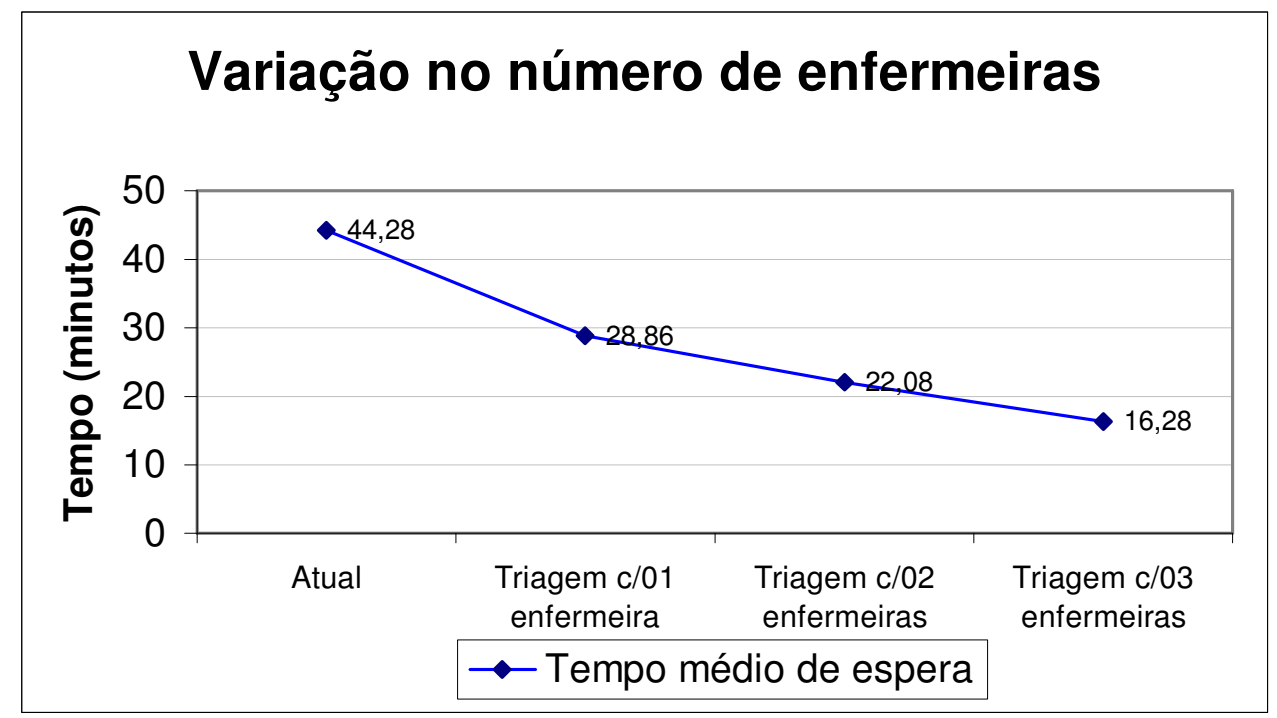

Figura 10 - Tempo médio de espera do paciente urgente por atendimento

Fonte: Sabbadini (2005, p. 179).

A partir dos resultados obtidos os gestores puderam tomar decisões quanto à alocação e a utilização de recursos humanos estratégicos, com o intuito de agilizar o atendimento aos pacientes de urgência.

\section{5- CONSIDERAÇÕES FINAIS}

A utilização da teoria das restrições no HMEHSG contribuiu para a melhoria no gerenciamento dos processos de tratamento aos pacientes com quadro de urgência naquela unidade de emergência hospitalar, através da identificação e do gerenciamento do gargalo no sistema.

A percepção antecipada por parte dos gestores, dos efeitos de uma mudança nos processos sobre o acompanhamento dos pacientes, mostrou-se importante no sentido de que estes puderam tomar decisões efetivas e realizar os ajustes necessários no sistema real.

A visão sistêmica obtida contribuiu para a compreensão do funcionamento das operações necessárias para o tratamento de pacientes, sendo possível identificar as interações entre as partes de cada um dos processos, os problemas potenciais e efetivos, e as oportunidades de melhorias contínuas.

O gerenciamento baseado na teoria das restrições assume uma dimensão estratégica num ambiente complexo em que se encontra o desafio de gerenciar permanentemente Revista Produção Online, Florianópolis, v.6, n.3,p.67, set./dez., 2006 
recursos especializados e de alto custo. Ao redirecionar as ações de melhorias nos recursos gargalo, pode-se ajustar a capacidade à demanda e atuar nos pontos do sistema em que os benefícios da alteração vão repercutir no aumento da capacidade global.

A redução do tempo entre o registro e o início do tratamento para o paciente urgente possui implicações importantes como a melhoria da assistência prestada a este paciente que passa a ter o acesso priorizado em função da gravidade do seu caso, iniciando-se mais rapidamente seu tratamento.

Os resultados obtidos demonstraram que com a triagem especializada reduz-se o tempo de espera do paciente urgente e conseqüentemente agiliza-se o início do seu tratamento.

Ao se alterar o direito de precedência na fila aumenta-se à eficiência do sistema em direção a meta da organização de atender com agilidade os pacientes com casos de urgência e reduzir o risco de seqüelas ou do agravamento do seu quadro clínico, tendo em vista um diagnóstico mais rápido que conseqüentemente possibilita o início mais ágil do tratamento ao paciente.

Um dos benefícios observados através da simulação refere-se à avaliação de mudanças nos processos. No HMEHSG a avaliação do cenário proposto mostrou que apesar de se adicionar uma atividade, a triagem especializada, obtém-se ganhos de produtividade na direção da meta da organização, possibilitando-se assim atender a um maior número de casos de pacientes de urgência, ao mesmo tempo em que se melhora a qualidade da atenção a estes.

Os experimentos realizados permitiram antecipar conseqüências de mudanças operacionais antes da sua implementação no sistema real, sem gerar ônus elevados, num setor complexo, com altos custos e elevados riscos, como é uma unidade de emergência hospitalar, que envolve o cuidado a vidas humanas.

As contribuições desta pesquisa estão relacionadas diretamente ao gerenciamento da capacidade dos serviços e na melhoria contínua dos serviços a serem prestados. $\mathrm{O}$ assunto não se esgota, devendo avançar, a partir do desenvolvimento de um estudo relativo ao comportamento da restrição no sistema. Segundo a teoria das restrições e o processo de focalização, uma vez que ocorre uma melhoria no gargalo, as restrições podem mudar de lugar no sistema.

Trabalhos futuros são indicados a partir de algumas observações deste estudo: a) O excessivo número de pacientes ambulatoriais na unidade de emergência é um fenômeno que deve ser estudado, avaliando as causas e analisando o fluxo desses pacientes a partir da rede 
primária. A aplicação da teoria das restrições para identificar e explorar gargalos na rede primária, de onde são provenientes pacientes ambulatoriais é uma oportunidade de pesquisa importante; b) A utilização da simulação computacional para avaliação de alternativas de melhoria na rede primária de saúde.

\section{REFERÊNCIAS}

BANKS, Jerry; CARSON, John S. Discrete-event system simulation. Prentice-Hall international series in industrial and system engineering. New Jersey: Prentice Hall, 1984.

CORREA, Henrique Luiz; GIANESI, Irineu G. N. Administração estratégica de serviços: operações para a satisfação do cliente. São Paulo: Atlas, 1994.

COX III, James F. Manual da teoria das restrições. Porto alegre : Bookman, 2002.

DE OLIVEIRA, M. J. F. A patient-oriented modeling of emergency admission system of a brasilian hospital. In EURO XIII, Glasgow, Jul, p. 19-22, 1994.

FITZSIMMONS, James A.; FITZSIMMONS, Mona J. Administração de serviços: operações, estratégia e tecnologia de informação. 2 ed. Porto Alegre: Bookman, 2000.

GOLDRATT, Elyahu M. What is this thing called Theory of Constraints and how should it be implemented? Massachusetts: North River Press, 1990.

GOLDRATT, Elyahu M. A Meta , 17ª edição, São Paulo: Educator, 1994.

GONÇALVES, Antônio Augusto. Gestão da capacidade de atendimento em hospitais de câncer. Dissertação (Doutorado em engenharia de produção). Rio de Janeiro : COPPE/UFRJ, 2004.

KERSHAW, R. Using TOC to cure healthcare problems. Management Acouting Quarterly, Spring, p. 22-28, 2000.

PHIPPS, Belinda. Hitting the bottleneck. Health Management. United Kingdon. 1999. Disponível em Goldratt Institute. ( www.goldratt.com ). Acesso em 20/jul/2004.

ROSSETTI, Manuel D.; TRZCINSKI, Gregory F.; SYVERUD, Scott A. Emergency department simulation and determination of optimal attending physician staffing schedules. In 1999 Winter Simulation Conference. Proceeding, Arizona 1999.

ROTSTEIN, Zeev; et al. Management by constraints: considering patient volume when adding medical staff to the emergency departament. IMAJ - International Medicine Australian Journal, v. 4, p. 171-174, march, 2002.

SABBADINI, F.S. Gerenciamento de restrições em hospital de emergência: um estudo de caso no Hospital Municipal Henrique Sérgio Gregori. Dissertação ( Mestrado em Administração e Desenvolvimento Empresarial ). Rio de Janeiro. UNESA, 2005. 
SCHRAGENHEIM, Eli. Management Dilemmas: The Theory of Constraints approach to problem identification and solutions. APICS Series on constraints management. Boca Raton:The St. Lucie Press, 1998.

VERGARA, Silvia Constant. Projetos e relatórios de pesquisa em administração. $6^{\mathrm{a}}$ ed. São Paulo : Atlas, 2005.

YIN, Robert. K. Estudo de caso: planejamento e métodos. 2. ed. Porto Alegre : Bookman, 2001. 